Department of Neurology, University of Erlangen

Nuremberg, Germany

J G Heckmann

C J G Lang

A Druschky

B Neundörfer

Department of Neurology,

Bezirkskrankenhaus

Ansbach, Germany

F Petruch

Department of Ophthalmology, University of Tübingen, Germany

C Erb

National Institutes of Health, Laboratory of Central Nervous System Studies, Bethesda, Maryland, USA

P Brown

Correspondence to: Dr Josef Georg Heckmann, Department of Neurology, University of

Erlangen-Nuremberg,

Schwabachanlage 6, D-91054

Erlangen, Germany.

Received 24 October 1996 and in revised form 7 March 1997

Accepted 14 March 1997

\title{
Transmission of Creutzfeldt-Jakob disease via a corneal transplant
}

\author{
J G Heckmann, C J G Lang, F Petruch, A Druschky, C Erb, P Brown, B Neundörfer
}

\begin{abstract}
A 45 year old woman is reported who initially presented with a cerebellar syndrome, severe ataxia, and dysarthria. She rapidly deteriorated to coma vigile with bilateral myoclonic jerks, flexion rigidity, and immobility necessitating complete nursing. Her EEG showed generalised slow activity and periodic biphasic and triphasic waves. The CSF concentration of neuron specific enolase was very high. Consequently the diagnosis of CreutzfeldtJakob disease was established. Eight months later she died of respiratory complications. Thirty years earlier the patient had undergone corneal transplantation for keratoconus. Review of the organ donor's hospital records showed that death was caused by intercurrent pneumonia subsequent to subacute spongiform encephalopathy confirmed by necropsy. In view of two previous case reports in the literature it is presumed that the cadaveric cornea was the source of transmission of Creutzfeldt-Jakob disease in this patient.
\end{abstract}

\section{(F Neurol Neurosurg Psychiatry 1997;63:388-390)}

Keywords: Creutzfeldt-Jakob disease; corneal graft; iatrogenic transmission

Since the first description of spongiform encephalopathy by Creutzfeldt and Jakob, much knowledge on the pathogenesis of Creutzfeldt-Jakob disease has been gained. The disease is classified into sporadic, familial, and iatrogenic types. Iatrogenic transmissions via human pituitary derived hormones, neurosurgical procedures, and implantation of lyophilised aura mater grafts are most commonly cited in the literature. Transmission of Creutzfeldt-Jakob disease by corneal grafting has been reported in only two instances. ${ }^{12}$ Here we describe the first European case of Creutzfeldt-Jakob disease after a corneal transplant.

\section{Case report}

A 45 year old woman was admitted to the neurological department of a district hospital complaining mainly of difficulties with walking and slightly slurred speech. A thorough neurological examination supplemented by CSF analysis, EEG, CCT, and MRT disclosed a cerebellar disease: a definite aetiology could not be established. The patient was transferred to the neurological department of a university hospital. She was alert and oriented to person, place, and date. Memory loss, particularly for recent events, was not noted. She had a wide based gait and was very ataxic, requiring assistance with walking. Truncal ataxia, cerebellar dysarthria, bilateral dysmetria on finger to nose and heel to knee pointing, and dysdiadochokinesia were also present. Other results of the neurological examination were unremarkable.

Investigations directed toward the possible presence of neoplastic, toxic, metabolic, inflammatory, and degenerative diseases of the cerebellum, including radiological contrast studies, were non-contributory.

Genetic analysis disclosed none of the pathogenic point or insert mutations that have been linked to familial forms of CreutzfeldtJakob disease. Methionine homozygosity was found at codon 129. There was no family history of Creutzfeldt-Jakob disease.

During her stay in the hospital, she deteriorated rapidly with severe dysarthria and dysphagia. She became demented, immobile with severe flexion rigidity in all four limbs and needed complete nursing. Myoclonic jerks were noted six weeks after admission, accentuated by noise and tactile stimulation.

Analysis of CSF disclosed a very high neuron specific enolase concentration $(117 \mathrm{ng} / \mathrm{ml}$; normal value $<20 \mathrm{ng} / \mathrm{ml}$ ). A repeat EEG showed generalised slow wave activity and periodic biphasic and triphasic discharges (figure). Myoclonic jerks were found during a period of six weeks and these then diminished. The patient died eight months after onset of her disease. A consent for necropsy could not be obtained.

We reviewed hospital records regarding the patient's history of two corneal transplantations in 1965 and 1982. In the files of the 1982 corneal transplantation, no remarks were made about the organ donor; in the 1965 files we discovered that the cornea had been taken from a 63 year old woman with a three month history of incoordination, memory loss, involuntary movements, and myoclonic jerks. The 
patient had deteriorated rapidly and, after intercurrent pneumonia, died one month later. Necropsy showed spongiform encephalopathy with characteristic change predominantly in the grey matter of the insula and the caudate nucleus; the frontal and occipital lobes were mildly affected. Unfortunately the histological specimen could not be reviewed. According to German law, the specimen has to be archived for at least 20 years, and then can be removed. In our case the specimen was discarded after 30 years consequent to a rebuilding of the neuropathological institute. However, the donor's original necropsy and medical reports could be reviewed describing CreutzfeldtJakob disease unequivocally.

\section{Discussion}

The clinical course, including cerebellar disorder, rapidly progressive mental deterioration, myoclonus, and very high enolase in CSF, and the typical EEG pattern are virtually diagnostic of Creutzfeldt-Jakob disease. ${ }^{3}$ Differential diagnosis of cerebellar disease in a middle aged patient including space occupying lesions, paraneoplastic syndrome, vitamin deficiency, alcoholic cerebellar degeneration, intoxication with drugs and heavy metals, encephalopathy due to JC virus, metabolic disorders such as hypothyroidism, or Refsum's disease were excluded by clinical, laboratory, and radiological examinations. Degenerative disorders such as olivopontocerebellar atrophy or cerebellar atrophy with late onset were excluded based on history, and neurological and instrumental findings.

Only two previous cases of Creutzfeldt-Jakob disease after corneal graft transplantation have been documented in the literature. In 1974 Duffy et $a l^{1}$ described a 55 year old woman who had received a corneal cadaveric graft and developed lethargy, nausea, and ataxia 18 months later. She deteriorated rapidly to a decorticate state and died eight months after onset of symptoms. Necropsy confirmed sus- pected Creutzfeldt-Jakob disease. Review showed that the donor cornea had been taken from a 55 year old man who had died of Creutzfeldt-Jakob disease confirmed by necropsy. A second case was reported in 1994 by Uchiyama et $a .^{2}$ A 63 year old otherwise healthy woman developed dysarthria, dysmetria, and dysdiadochokinesia 15 months after corneal transplantation. Neurologically she deteriorated by difficulty in walking, swallowing, hypereactive reflexes, myoclonic jerks, and paranoid hallucinations. She died three years and four months after onset of disease. Necropsy showed typical Creutzfeldt-Jakob disease. Regarding the "incubation period" of 15 months after corneal transplantation, similar to the case reported by Duffy et al, ${ }^{1}$ the authors assumed a strong link between the Creutzfeldt-Jakob disease and the previous cornea transplantation. However, exact data about the organ donor in this case were not given.

In our case we also presume a link between the illness and the precedent corneal transplantation for several reasons. Firstly, the patient received a corneal cadaveric graft at a time when the possibility of transmission of Creutzfeldt-Jakob disease via corneal tissue was still unknown. Thus donor patients with potential Creutzfeldt-Jakob disease were not regularly excluded from organ donation yielding the risk of transmission higher than today. Secondly, the donor had Creutzfeldt-Jakob disease verified by necropsy. Thirdly, in iatrogenic Creutzfeldt-Jakob disease the latency from inoculation to onset of disease varies from 15 months to a maximum of 30 years, ${ }^{45}$ the same period that elapsed in our case after keratoplasty. Perhaps this phenomenon depends on the mode of inoculation or is genetically determined. In the two previously described iatrogenic cases of Creutzfeldt-Jakob disease after corneal tranplant the incubation period was very short, 15 and 18 months after operation. In our patient the incubation period was, however,

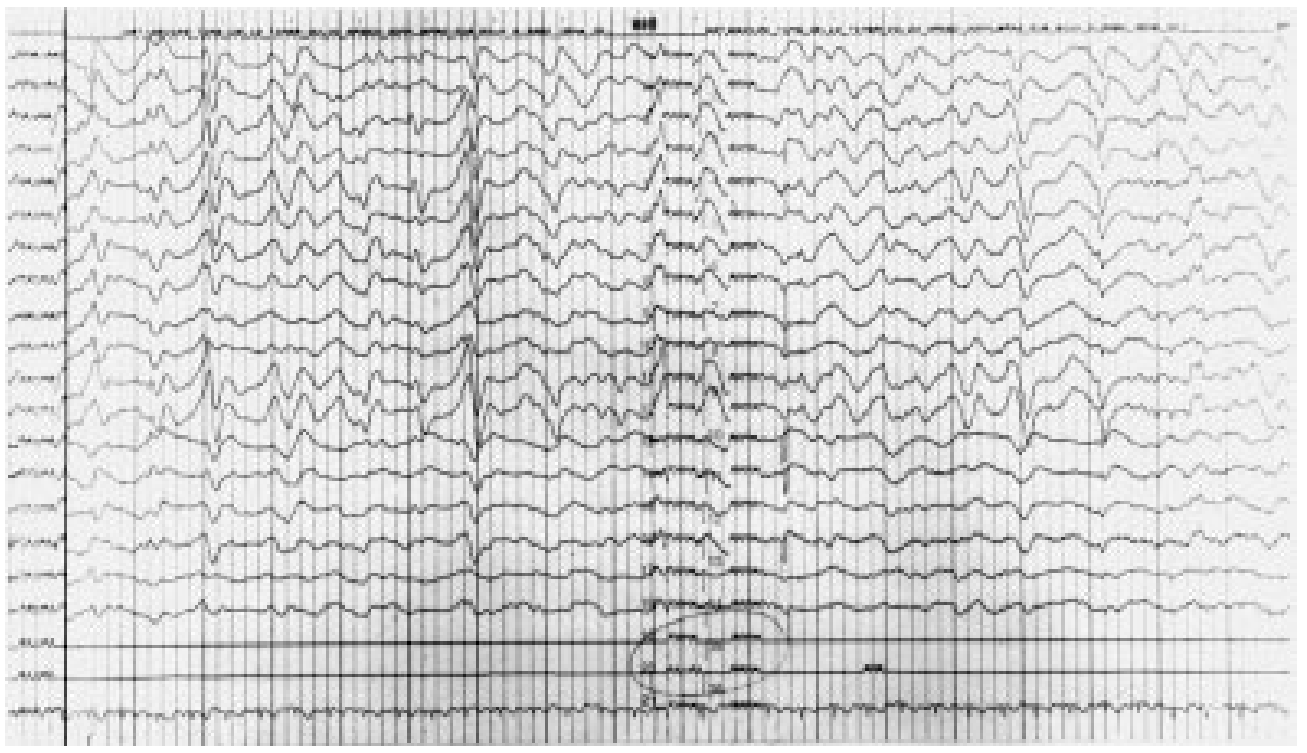

EEG of the patient two weeks after rapid mental deterioration. The unipolar leads (top to bottom: right and left side alternatively) show pronounced slowing of background activity and repeated triphasic discharges. 
unusually lengthy. An explanation of this feature is not evident, but may depend on a "strain effect" of the Creutzfeldt-Jakob disease agent. Fourthly, in iatrogenic Creutzfeldt-Jakob disease the illness may start as a predominantly cerebellar rather than cementing syndrome. ${ }^{45}$ Both previously reported patients who developed Creutzfeldt-Jakob disease after corneal transplantation showed initially cerebellar signs such as ataxia and dysarthria, as was also shown in our case. Finally, methionine homozygosity at the polymorphic codon 129 , which enhances susceptibility to iatrogenic infection, was shown genetically.

In summary, we think that the case reported here represents a third instance of iatrogenically transmitted Creutzfeldt-Jakob disease after corneal transplantation. Our aim is to draw attention to this possible fatal late complication after corneal transplantation stressing the need for careful donor selection.
We thank Professor Dr HA Kretschmar, Department of Neuropathology of the University of Göttingen, Germany, for the genetic analysis, Professor Dr G Koniszewski, Department of Ophthalmology of the City Hospital Nurnberg for reviewing the medical records, Dr H Murata and Mr K Fukai for translating original reference 2, and $\mathrm{Mr} \mathrm{A}$ Lawrence for linguistic advice.

1 Duffy P, Wolf J, Collins G, DeVoe AG, Sreeten B, Cowen D. Possible person-to-person transmission of CreutzfeldtJakob disease. N Engl f Med 1974;290:692-3.

2 Uchiyama K, Ishida C, Yago S, Kurumaya H, Kitamoto T. An autopsy case of Creutzfeldt-Jacob disease associated with corneal transplantation. Dementia 1994;8:466-73. (In with corneal

3 Brown P, Gibbs CJ, Rodgers-Johnson P, Asher DM, Sulima MP, Bacote A, Goldfarb LG, Gajdusek DC. Human spongiform encephalopathy: The National Institutes of Health series of 300 cases of experimentally transmitted disease. Ann Neurol 1994;35:513-29.

4 Brown P, Cervenakova L, Goldfarb LG, McCombie WR, Rubenstein R, Will RG, et al. Iatrogenic Creutzfeldt-Jakob disease: an example of the interplay between ancient genes and modern medicine. Neurology 1994;44:291-3.

5 Brown P. Enviromental causes of human spongiform encephalopathy. In:H Baker, R Ridley, eds. Methods in molecular medicine: prion diseases. Totowa, New Jersey: Humana Press, 1996:139-54. 